\title{
APPENDIX C: RECOMMENDATIONS OF BEST PRACTICES
}

- Have a solid knowledge of the inner workings of the chosen platform.

- All users should know the normal functions; superusers or practice champions should have advanced knowledge.

- Practice workflow should be implemented prior to go-live.

- Establish a back-up plan in case the primary option for digital health becomes unavailable.

- Implement appropriate protocols should emergent situations occur.

- Implement screening questions (COVID-19 or other infectious disease-related).

- All records of digital health encounters should be kept as they would for in-person evaluations.

- Be consistent in updating the practice's knowledge of rules, regulations, and laws.

- Be aware of the current climate with telemedicine. 
- Have staff properly assess who is interested in digital services versus who prefers in-person evaluations.

- Be conscientious when deciding upon a self-pay rate. Should balance out cost to cover services rendered while being affordable for the patient. 